Temperature and composition profile during double-track laser cladding of $\mathrm{H} 13$ tool steel

This article has been downloaded from IOPscience. Please scroll down to see the full text article.

2010 J. Phys. D: Appl. Phys. 43015502

(http://iopscience.iop.org/0022-3727/43/1/015502)

The Table of Contents and more related content is available

Download details:

IP Address: 159.226.231.96

The article was downloaded on 24/12/2009 at 06:45

Please note that terms and conditions apply. 


\title{
Temperature and composition profile during double-track laser cladding of H13 tool steel
}

\author{
X He${ }^{1}, \mathbf{G ~ Y u}{ }^{1}$ and $\mathrm{J}$ Mazumder ${ }^{2}$ \\ ${ }^{1}$ Key Laboratory of Mechanics in Advanced Manufacturing, Institute of Mechanics, Chinese Academy \\ of Sciences, Beijing 100190, People's Republic of China \\ ${ }^{2}$ Center for Laser-Aided Intelligent Manufacturing, University of Michigan, Ann Arbor, MI 48109, USA
}

Received 28 July 2009, in final form 29 October 2009

Published 7 December 2009

Online at stacks.iop.org/JPhysD/43/015502

\begin{abstract}
Multi-track laser cladding is now applied commercially in a range of industries such as automotive, mining and aerospace due to its diversified potential for material processing. The knowledge of temperature, velocity and composition distribution history is essential for a better understanding of the process and subsequent microstructure evolution and properties. Numerical simulation not only helps to understand the complex physical phenomena and underlying principles involved in this process, but it can also be used in the process prediction and system control. The double-track coaxial laser cladding with H13 tool steel powder injection is simulated using a comprehensive three-dimensional model, based on the mass, momentum, energy conservation and solute transport equation. Some important physical phenomena, such as heat transfer, phase changes, mass addition and fluid flow, are taken into account in the calculation. The physical properties for a mixture of solid and liquid phase are defined by treating it as a continuum media. The velocity of the laser beam during the transition between two tracks is considered. The evolution of temperature and composition of different monitoring locations is simulated.
\end{abstract}

(Some figures in this article are in colour only in the electronic version)

\section{Introduction}

Multi-track laser cladding with coaxial powder injection has received more and more attention due to its diversified potential for computer controlled direct metal deposition (DMD). Coaxial powder injection enables a DMD system to move in any direction as needed based on the computer aided design/computer aided manufacturing (CAD/CAM) program to create a three-dimensional object. Unlike single-track cladding, the materials in each track will undergo successive thermal cycles as the laser beam moves to the other tracks in multi-track cladding. As a result, the phase transformations activated by reheating or remelting lead to the modification of the microstructure and properties of the materials. Since the thermal cycles differ from point to point, the material will present a complex and heterogeneous microstructure and property after laser cladding.
There are a large number of variables involved in the laser cladding process, including laser processing parameters, thermo-physical variables of substrate and powder. Selection of inappropriate combinations can result in problems such as material porosity, excessive dilution of substrate and poor catchment efficiency of powder. High quality deposition needs a lot of trial experiments. However, systematic experimental investigations using statistical design of experiment (DOE) appear to be a time-consuming way due to the complexity and nonlinearity of the laser cladding process. Moreover, in situ measurements near the liquid pool are difficult due to the localized nature of laser heating, small melt pool size, rapid heating and cooling. With considerable progress made in computer hardware and software, numerical simulation becomes an efficient way to aid in the quantitative study of laser aided deposition and obtain necessary information for the prediction of morphologies and thermal field induced during the laser cladding process. 
Because of demanding longer computer run-time and bigger data storage space for multi-track laser cladding, most numerical models for laser cladding focused on single-track laser processing [1-11]. However, with the rapid development of multi-track laser processing, a deeper understanding of temperature field and its effects on the thermo-physical properties of materials is more pressing. Colombo et al [12] simulated the temperature evolution on the surface of a work piece for the double-track laser surface heating process that did not lead to material melting or evaporation, in the situations of overlapping, non-overlapping and of a gap left between the two parallel tracks of the laser beam. Ghosh and Choi $[13,14]$ simulated temperature, cooling rate distribution and phase transformation by a simple three-dimensional thermokinetic finite element model for a double-track laser cladding process. Lakhkar et al [15] developed a coupled heat transfer-hardening-tempering model to predict the hardness and phases in the back-tempered zones during multi-track laser hardening of AISI 4140 steel. The model can be used to predict the volume fraction of martensite that has been tempered and the corresponding hardness values. Li et al [16] calculated the temperature field of multi-layer laser cladding through the finite-difference method by using the measured data as the boundary conditions. The calculation indicated that the temperature gradient in the laser melted pool was about $10^{5}-10^{6} \mathrm{~K} \mathrm{~m}^{-1}$. A three-dimensional thermokinetic finite element model of laser powder deposition of tool steels was developed by Costa et al $[17,18]$ to simulate the temperature, volume fraction of phases and average hardness. It was shown that the temperature field depended significantly on the size of the substrate. Jendrzejewski et al $[19,20]$ simulated the temperature distribution for multi-layer structures prepared by direct laser remelting of metal powders. A three-dimensional finite element model was developed by Wang and Felicelli [21] to simulate the laser engineered net shaping rapid fabrication process of a stainless steel 410 plate. The model was used to analyse the temperature distribution, molten pool size, and volume fraction of the solid phases formed at different traverse speeds. Kelly and Kampe [22] developed a numerical thermal model based on the implicit finite-difference technique to simulate laser metal deposition processes. The results showed that the heating rate and peak thermal gradient within a deposited layer had the same trend as the peak temperature after two layers were deposited. The cooling rate in a newly deposited layer decreased as the number of layer additions increased. In all of the above simulations, the important factor during the multi-track DMD process, the liquid flow in the melt pool was ignored.

In this study, a mathematical three-dimensional model was developed to simulate the temperature and composition distribution during the laser cladding process of $\mathrm{H} 13$ tool steel. Heat transfer, fluid flow, melting and solidification, phase changes, and laser beam velocity during the transition process between two tracks are considered. Due to longer time and bigger storage space for the multi-track laser cladding process, the simulation results shown in this paper are mainly focused on the double-track laser cladding process.

\section{Mathematical modeling}

\subsection{Assumptions}

The comprehensive 3D mathematical model to simulate the temperature and composition profile during the double-track DMD process is based on the assumption of an incompressible, laminar and Newtonian liquid flow. Besides, the following assumptions are made in order to simplify the calculations:

(1) The thermo-physical properties and absorption coefficient of laser are assumed to be temperature independent. However, the numerical model is capable of easily incorporating the temperature dependent thermo-physical properties.

(2) The thermo-physical properties of powder are considered the same as those of the substrate.

(3) The input heat from laser is assumed to have a Gaussian distribution. The beam radius and profile remain constant along the $z$ direction.

(4) The velocity of laser moving from the first to the second track is assumed to be half of the laser scanning speed.

(5) The shape of powder particles is spherical. The powders falling in the region of liquid surface gets melted immediately.

The last assumption is based on the model of spherical particle melting [23]. The melting of a spherical steel particle bathed in the environment at constant melting temperature would be complete in $10^{-5} \mathrm{~s}$ for a $10 \mu \mathrm{m}$ diameter particle. Hence, the powders falling in the region of liquid surface can be considered to melt immediately.

\subsection{Governing equations}

The basic transport phenomena, mass, momentum, energy and species transfer can be expressed by the following governing equations.

Continuity equation:

$$
\frac{\partial \rho}{\partial t}+\nabla \cdot(\rho \boldsymbol{u})=0
$$

Momentum equations:

$$
\begin{aligned}
& \frac{\partial(\rho u)}{\partial t}+\nabla \cdot(\rho \boldsymbol{u} u)=\nabla \cdot(\mu \nabla u)-\frac{\mu_{1}}{K} \frac{\rho}{\rho_{1}} u-\frac{\partial p}{\partial x}, \\
& \frac{\partial(\rho v)}{\partial t}+\nabla \cdot(\rho \boldsymbol{u} v)=\nabla \cdot(\mu \nabla v)-\frac{\mu_{1}}{K} \frac{\rho}{\rho_{1}} v-\frac{\partial p}{\partial y} . \\
& \frac{\partial(\rho w)}{\partial t}+\nabla \cdot(\rho \boldsymbol{u} w) \\
& \quad=\nabla \cdot(\mu \nabla w)-\frac{\mu_{1}}{K} \frac{\rho}{\rho_{1}} w-\frac{\partial p}{\partial z}+\rho g \beta\left(T-T_{0}\right) .
\end{aligned}
$$

Energy equation:

$$
\begin{aligned}
& \frac{\partial\left(\rho C_{p} T\right)}{\partial t}+\boldsymbol{u} \cdot \nabla\left(\rho C_{p} T\right) \\
& \quad=\nabla \cdot(k \nabla T)-\frac{\partial\left(\rho f_{\mathrm{s}} \mathcal{L}\right)}{\partial t}+\frac{\partial\left(\rho f_{\mathrm{s}} \Delta \bar{C}_{p} T\right)}{\partial t} .
\end{aligned}
$$




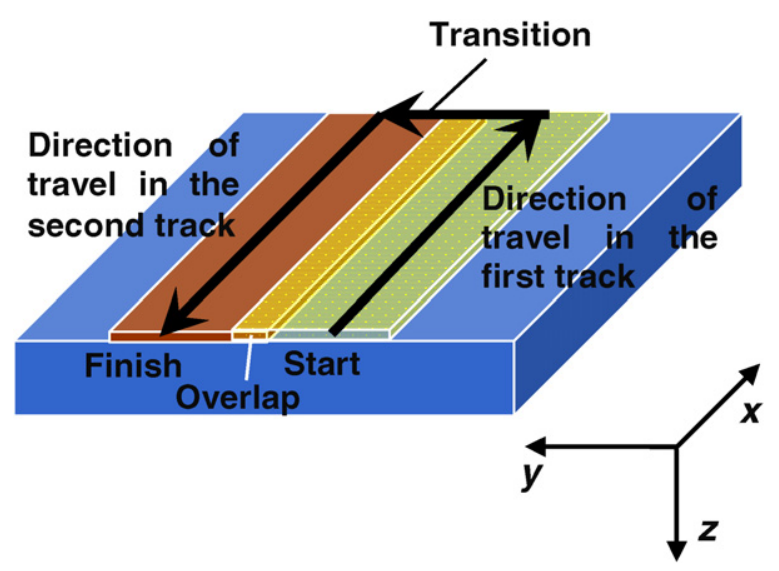

Figure 1. Schematic of the double-track laser cladding process.
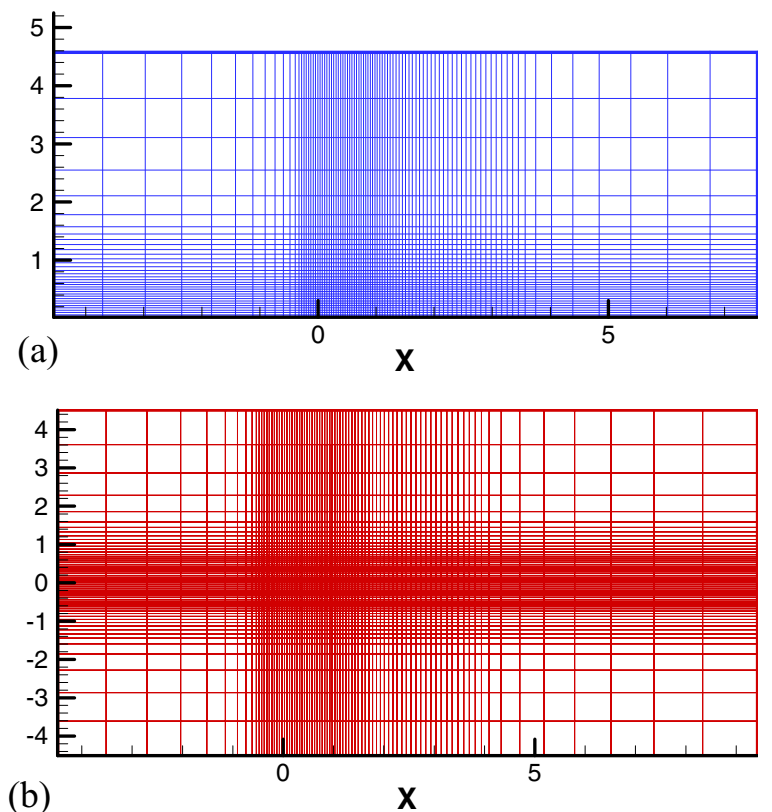

Figure 2. Grid system of the top surface used for $(a)$ single-track and $(b)$ double-track laser cladding process.

Concentration equation:

$$
\begin{aligned}
\frac{\partial(\rho c)}{\partial t} & +\nabla \cdot(\rho \boldsymbol{u c})=\nabla \cdot(\rho D \nabla c)+\nabla \cdot\left[\rho D \nabla\left(c_{1}-c\right)\right] \\
& -\nabla \cdot\left[\rho f_{\mathrm{s}}\left(c_{1}-c_{\mathrm{s}}\right) \boldsymbol{u}\right],
\end{aligned}
$$

where $\rho$ is the density, $t$ is the time, $u, v, w$ are the liquid velocities along the $x, y, z$ directions, respectively, $\mu$ is the viscosity and $p$ is the pressure. The second term in the righthand side of equations (2)-(4) is the Darcy term, representing the damping force when fluid is passing through a porous media and $K$ is the isotropic permeability, which can be expressed by Kozeny-Carman equation [24]. The last term in equation (4) is the buoyancy force, $\beta$ is the thermal expansion coefficient. $C_{p}$ is the specific heat, $T$ is the temperature, $k$ is the thermal conductivity, $f$ is the mass fraction, $c$ is the solute concentration and $D$ is the mass diffusivity. The subscript s and 1 denote the solid and liquid phases, respectively. The last two terms in equation (6) account for the species flux due to relative phase diffusion and motion, respectively. They are
Table 1. Data used for calculations.

\begin{tabular}{ll}
\hline Property & Value \\
\hline Absorption coefficient & 0.1 \\
Liquid density $\left(\mathrm{kg} \mathrm{m}^{-3}\right)$ & 6518.5 \\
Solid density $\left(\mathrm{kg} \mathrm{m}^{-3}\right)$ & 7870 \\
Liquid viscosity $\left(\mathrm{N} \mathrm{s} \mathrm{m}^{-2}\right)$ & 0.005 \\
Solidus temperature $(\mathrm{K})$ & 1776 \\
Liquidus temperature $(\mathrm{K})$ & 1800.4 \\
Latent heat of fusion $\left(\mathrm{J} \mathrm{kg}^{-1}\right)$ & $2.72 \times 10^{5}$ \\
Solid specific heat $\left(\mathrm{J} \mathrm{kg}^{-1} \mathrm{~K}^{-1}\right)$ & 658.63 \\
Liquid specific heat $\left(\mathrm{J} \mathrm{kg}^{-1} \mathrm{~K}^{-1}\right)$ & 804.03 \\
Solid thermal conductivity $\left(\mathrm{W} \mathrm{m}^{-1} \mathrm{~K}^{-1}\right)$ & 40.96 \\
Liquid thermal conductivity $\left(\mathrm{W} \mathrm{m}^{-1} \mathrm{~K}^{-1}\right)$ & 43.99 \\
Surface tension coefficient $\left(\mathrm{N} \mathrm{m}^{-1} \mathrm{~K}^{-1}\right)$ & -0.00049 \\
Mass diffusivity $\left(\mathrm{m}^{2} \mathrm{~s}^{-1}\right)$ & $3.0 \times 10^{-8}$ \\
Thermal expansion coefficient $\left(\mathrm{K}^{-1}\right)$ & $1.45 \times 10^{-4}$ \\
\hline
\end{tabular}

equivalent to zero when applied in pure liquid or solid phase. In equation $(5), \mathcal{L}$ is defined as

$$
\mathcal{L}=\left(\bar{C}_{p \mathrm{~s}}-\bar{C}_{p 1}\right) T_{\mathrm{e}}+L_{m}=\Delta \bar{C}_{p} T_{\mathrm{e}}+L_{m},
$$

where $L_{m}$ is the latent heat of fusion, $T_{\mathrm{e}}$ is the eutectic temperature and $\bar{C}_{p}$ is the average specific heat.

In this study, the mushy zone, containing both the solid and liquid phases, is treated as a continuum media. A method developed by Bennon and Incropera [25, 26] is adopted for a binary $\mathrm{S} / \mathrm{L}$ phase system. The physical properties for a mixture of solid and liquid phase are defined as follows:

$$
\begin{gathered}
\rho=g_{\mathrm{s}} \rho_{\mathrm{s}}+g_{1} \rho_{\mathrm{l}}, \\
k=\left(\frac{g_{\mathrm{s}}}{k_{\mathrm{s}}}+\frac{g_{1}}{k_{\mathrm{l}}}\right)^{-1}, \\
C_{p}=f_{\mathrm{s}} C_{p \mathrm{~s}}+f_{1} C_{p l}, \\
c=f_{\mathrm{s}} c_{\mathrm{s}}+f_{1} c_{1}, \\
D=f_{1} D_{1},
\end{gathered}
$$

where $g$ is the volume fraction.

\subsection{Boundary conditions}

The boundary condition at the L/G interface for energy equation can be formulated as

$$
\begin{aligned}
q_{\mathrm{L} / \mathrm{G}}= & \frac{2 Q^{\prime} \alpha}{\pi r_{\mathrm{b}}^{2}} \exp \left(\frac{-2 r^{2}}{r_{\mathrm{b}}^{2}}\right)+q_{\mathrm{p}}-h_{\mathrm{c}}\left(T-T_{0}\right) \\
& -\sigma \varepsilon\left(T^{4}-T_{0}^{4}\right)-\rho_{\mathrm{l}} L_{\mathrm{v}} F_{\mathrm{e}} .
\end{aligned}
$$

The five terms in the right-hand side represent the energy by the laser with Gaussian distribution, the addition of heated powder, and the heat loss by convection, radiation and evaporation, respectively. $Q^{\prime}$ is the attenuated laser power (as calculated in [1]), $\alpha$ is the laser absorption coefficient, $r_{\mathrm{b}}$ is the effective beam radius, $r$ is the radial distance to the laser beam, $h_{\mathrm{c}}$ is the heat transfer coefficient, $\sigma$ is the Stefan-Boltzmann constant, $\varepsilon$ is the emissivity, $T_{0}$ is the ambient temperature. $L_{\mathrm{v}}$ is the latent heat of evaporation and $F_{\mathrm{e}}$ is the evaporation flux. 
Table 2. Comparison between calculated and experimental cladding size.

\begin{tabular}{|c|c|c|c|c|c|c|c|c|}
\hline \multirow{2}{*}{$\begin{array}{l}\text { Laser power } \\
\text { (W) }\end{array}$} & \multirow{2}{*}{$\begin{array}{l}\text { Scanning speed } \\
\left(\mathrm{mm} \mathrm{min}^{-1}\right)\end{array}$} & \multirow{2}{*}{$\begin{array}{l}\text { Beam size } \\
(\mathrm{mm})\end{array}$} & \multicolumn{2}{|c|}{ Cladding width $(\mathrm{mm})$} & \multicolumn{2}{|c|}{ Cladding depth (mm) } & \multicolumn{2}{|c|}{ Cladding height $(\mathrm{mm})$} \\
\hline & & & $\operatorname{Exp}^{a}$ & $\mathrm{Cal}^{\mathrm{a}}$ & $\operatorname{Exp}^{a}$ & $\mathrm{Cal}^{\mathrm{a}}$ & $\operatorname{Exp}^{\mathrm{a}}$ & $\mathrm{Cal}^{\mathrm{a}}$ \\
\hline \multirow[t]{4}{*}{1200} & 300 & 1.2 & 1.546 & 1.484 & 0.093 & 0.076 & 0.177 & 0.126 \\
\hline & & & 1.638 & & 0.082 & & 0.179 & \\
\hline & & & 1.520 & & 0.120 & & 0.151 & \\
\hline & & & 1.501 & & 0.107 & & 0.162 & \\
\hline 1900 & 200 & 1.8 & 2.97 & 2.893 & 0.108 & 0.159 & 0.331 & 0.354 \\
\hline
\end{tabular}

a 'Exp' and 'Cal' indicate experimentally measured and calculated results, respectively.

The extra energy brought by the addition of heated powder can be expressed as

$q_{p}= \begin{cases}F_{\mathrm{p}} \rho_{\mathrm{l}}\left[C_{p \mathrm{~s}}\left(T_{m}-T_{0}\right)+L_{m}+C_{p \mathrm{l}}\left(T-T_{m}\right)\right] & T>T_{m}, \\ F_{\mathrm{p}} \rho_{\mathrm{l}}\left[C_{p \mathrm{~s}}\left(T_{m}-T_{0}\right)+L_{m} f_{\mathrm{l}}-f_{\mathrm{s}} L_{m}\right] & T=T_{m}, \\ F_{\mathrm{p}} \rho_{\mathrm{s}}\left[C_{p \mathrm{~s}}\left(T-T_{0}\right)-L_{m}-C_{p \mathrm{~s}}\left(T_{m}-T\right)\right] & T<T_{m},\end{cases}$

where $F_{\mathrm{p}}$ is the free surface growth velocity due to powder addition.

Two forces are considered at the L/G interface-the capillary and thermocapillary forces. The capillary force acts in the normal direction due to the interface curvature and surface tension. The thermocapillary force acts in the tangential direction of the liquid free surface, and is caused by the surface temperature gradient. These forces can be formulated as

$$
F_{\mathrm{L} / \mathrm{G}}=\sigma \boldsymbol{n}^{*} \kappa-\nabla_{\mathrm{s}} T \frac{\mathrm{d} \sigma}{\mathrm{d} T}
$$

where $\sigma$ is the surface tension, $n^{*}$ is the normal of the surface pointing inwards to the liquid phase, $\kappa$ is the curvature.

During an equilibrium solidification process, solute atoms are rejected from the solidification front and diffuse into the liquid phase. The boundary condition at the solidification interface can be given by the gradient of solute concentration on the liquid side:

$$
-D_{1} \frac{\partial c_{1}}{\partial n}=\left(1-k_{\mathrm{p}}\right) v_{n} c_{1}
$$

where $n$ is the direction of the outward normal, $k_{\mathrm{p}}$ is the equilibrium partition coefficient and $v_{n}$ is the interface velocity in that direction. As the powder with a known composition of mixed material $\left(c_{p}\right)$ is continuously falling onto the molten pool surface, the boundary condition at the liquid/gas interface is

$$
\left.c\right|_{\varphi=0}=c_{p},
$$

where $\varphi$ is the level-set function [1]. For the liquid-gas interface, $\varphi=0$.

\subsection{Grid spacings and time step}

The schematic of the double-track laser cladding process is shown in figure 1 . The laser moves along the $x$ direction until it completes the first track. There is a transition process after the first track. Then the laser begins the second track and returns. There is an overlap region between two tracks.

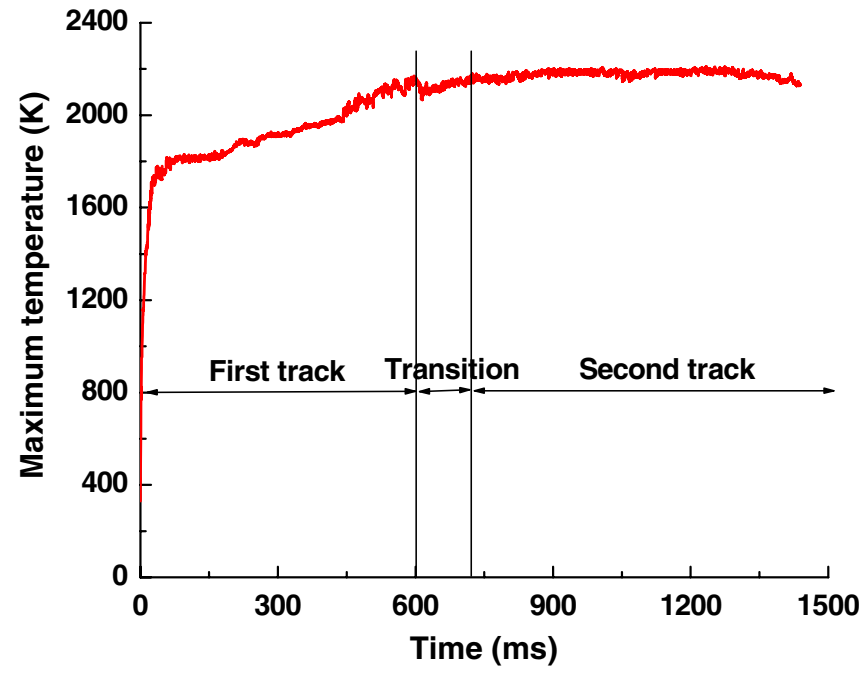

Figure 3. Variation of maximum temperature in computational domain with time. Laser power: $1200 \mathrm{~W}$, beam diameter: $1.2 \mathrm{~mm}$, scanning speed: $300 \mathrm{~mm} \mathrm{~min}^{-1}$ and powder flow rate: $8 \mathrm{~g} \mathrm{~min}{ }^{-1}$.

The authors' previous work focused on the single-track laser cladding process [1]. The temperature and velocity fields were essentially symmetrical with respect to the laser moving direction due to the nature of heat distribution. Only half domain was considered in order to save computation time. However, for the double-track laser cladding process, the temperature and velocity fields are not axis-symmetrical. As a result, the entire domain has to be considered. Figure 2 shows the comparison between the grid system of the top surface used for the single-track and double-track laser cladding process. The top surface in this paper means the surface with the $z$ coordinate of 0 .

Accurate calculation of temperature and velocity fields, concentration distribution requires use of a very fine grid system. The basic grid system used in the calculation for the double-track laser cladding process contains $83 \times 69 \times 45$ grid points. Spatially non-uniform grids are used for maximum resolution of variables. A finer grid spacing is used near the heat source. The minimum grid space along the $x, y$ and $z$ directions are about $50 \mu \mathrm{m}, 47.6 \mu \mathrm{m}$ and $23.8 \mu \mathrm{m}$, respectively.

Typically, the time step should be very small (less than $10^{-5} \mathrm{~s}$ ) to satisfy the convergence criteria requested by governing equations, as well as the movement of liquid free surface( $\mathrm{L} / \mathrm{G}$ interface). The maximum displacement of the interface at one time step should be less than the minimum grid spacing. 

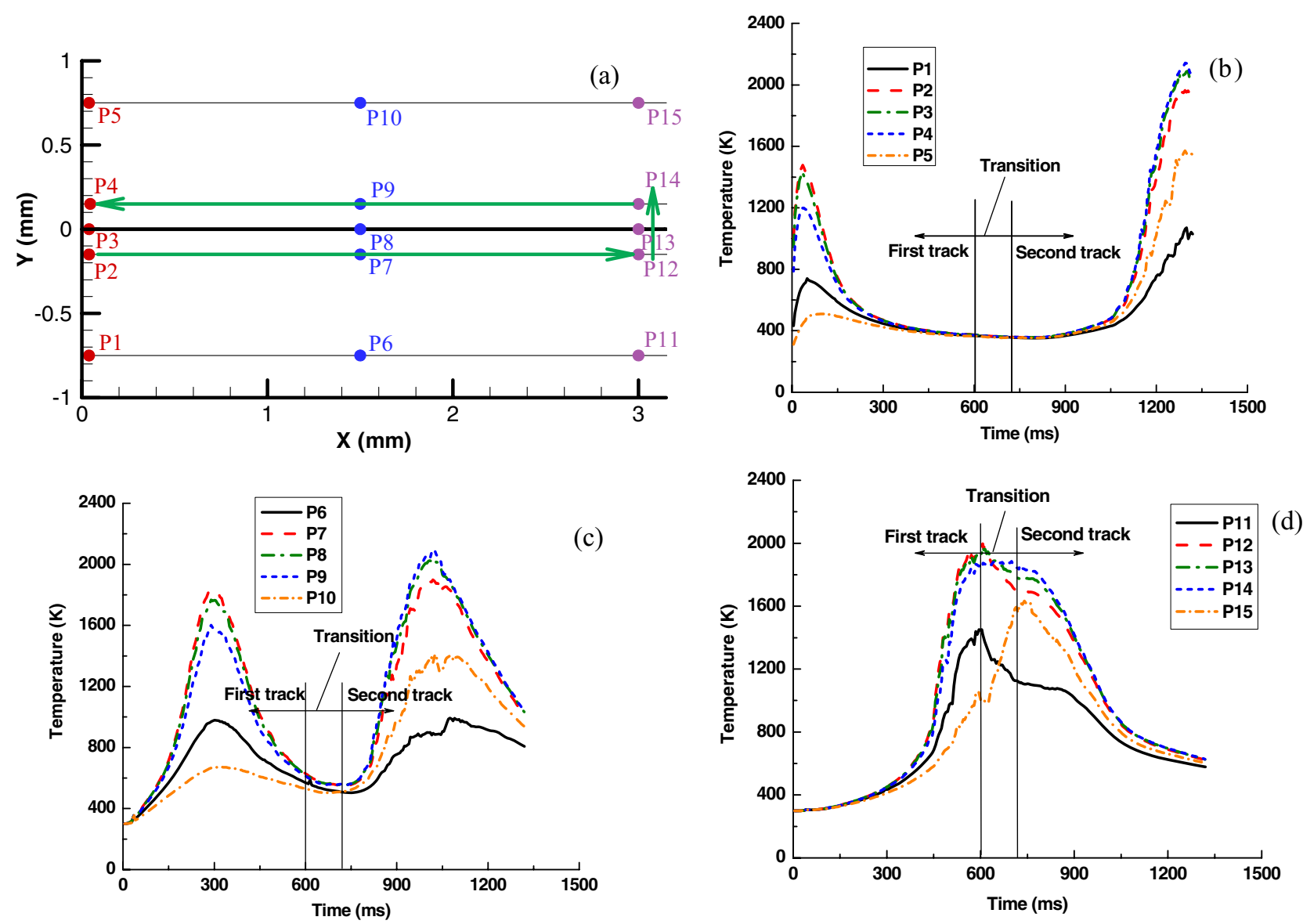

Figure 4. (a) Schematic of different points and thermal cycles of different locations of $(b)$ first group, $(c)$ second group and $(d)$ third group. Laser power: $1200 \mathrm{~W}$, beam diameter: $1.2 \mathrm{~mm}$, scanning speed: $300 \mathrm{~mm} \mathrm{~min}^{-1}$ and powder flow rate: $8 \mathrm{~g} \mathrm{~min}^{-1}$.

With appropriate boundary conditions, the governing equations are discretized with the implicit control volume finite-difference method in three dimensions and then solved using a symmetrically coupled Gauss Seidel (SCGS) point relaxation scheme [27]. More details on interaction between laser and H13 powder, the level-set method to track the evolution of the liquid-gas interface, discretization of governing equations and convergence criteria have been described in the authors' previous paper [1] and are not repeated here.

\section{Results}

\subsection{Validation of the model}

The laser cladding experiments were performed for ANSI H13 tool steel as the powder, deposited on the low carbon steel substrate. The absorptivity and thermo-physical properties of material used for calculations are presented in table $1[10,28]$. The calculated and experimental cladding size is compared in table 2. For the first parameter set, the experiments with the same processing parameters were performed four times. The discrepancy between the calculated and experimental results may be caused by not considering the effect of temperature on the thermo-physical properties and absorption coefficient of substrate and powder. For the calculation of cladding height, the discrepancy between the calculated and experimental results is potentially due to the variance of the powder concentration distribution in the real process from the assumed Gaussian distribution in the model.

\subsection{Temperature profile}

3.2.1. Maximum temperature. The simulation of doubletrack laser cladding was performed for ANSI H13 tool steel powder deposited on low carbon steel, with $75 \%$ overlap. Figure 3 shows the maximum temperature in the entire computational domain as a function of time. Generally, the location where laser density is the highest has the maximum temperature. Some useful information can be obtained from the evolution of the maximum temperature, such as the formation time of the melt pool or the time when the melt pool reaches the steady state. In the initial period, the temperature increases very quickly due to sudden heat input from laser. After about $36 \mathrm{~ms}$, the maximum temperature exceeds the solidus temperature and the liquid pool begins to form. At the time of $600 \mathrm{~ms}$, the laser completes the first track and begins the transition process. It is shown that the maximum temperature does not change a lot for the transition process and second track.

3.2.2. Thermal cycle of points. Figure 4 shows the thermal cycles of different monitoring locations at the top surface. The 
three arrows shown in figure 4(a) represent the laser moving direction. The thermal cycles of 15 selected points are shown in figures $4(b)-(d)$. The 15 points are divided into three groups. The points in the same group have the same value for the $x$ coordinate.

After the laser is irradiated, the substrate absorbs the energy from the laser and the temperature increases quickly until the maximum temperature is reached. For various monitoring locations, the temperature, heating and cooling rates decrease with increasing distance from the laser beam.

For the first group with the points $\mathrm{p} 1$ to $\mathrm{p} 5$, there are two peaks for every thermal cycle curve since the laser gets close to these points twice in its entire travel (shown in figure $4(b)$ ). Points p2 and p5 are the closest and farthest point to the laser beam during the first track. As a result, they have the highest and lowest temperature, respectively. With the laser moving away, the temperatures of these points decrease until the second track begins. All of these points do not reach the melting temperature for the first track. During the transition process, these points have similar temperature because of being far from the laser. After the laser begins the second track, the temperatures of these points increase due to being closer to the laser beam.

For the second group with the points p6 to p10 (shown in figure $4(c)$ ), because they are farther from the starting point of the laser beam, it takes a longer time to reach the peak temperature. Similar to the previous group, there are two peaks for points $\mathrm{p} 6$ to $\mathrm{p} 10$. For point $\mathrm{p} 8$, with the similar geometric position to the first and second track, the peak temperature for the second track is higher than the previous peak. This also happens to points $\mathrm{p} 6$ and p7, even though the distance to the second track was farther than that to the first track. This is caused by the preheating due to the first track of the laser.

For the third group with points p11 to p15 (shown in figure $4(d)$ ), it takes them even longer time to reach the peak temperature than points $\mathrm{p} 1$ to $\mathrm{p} 10$. Unlike the other two groups, the thermal cycle curves of the points of the third group has only one peak since laser gets close to these points only once in its entire travel.

3.2.3. Temperature distribution of lines. Figure 5 shows temperature distribution along the $x, y$ and $z$ directions at four typical times. $600 \mathrm{~ms}$ and $720 \mathrm{~ms}$ are the starting and ending time of the transition process, respectively. At times of $300 \mathrm{~ms}$ and $1020 \mathrm{~ms}$, the position of the laser has the same value for the $x$ coordinate. Figure 5( $a$ ) shows the temperature distribution along the $x$ direction, the intersection of the symmetric plane and the top surface. Figure $5(b)$ shows the temperature distribution along the $y$ direction, the intersection of the top surface and the plane with the $x$ coordinate of $2.25 \mathrm{~mm}$, as shown in the small figure. For the curves at times of $300 \mathrm{~ms}$ and $1020 \mathrm{~ms}$, the peak temperature for $1020 \mathrm{~ms}$ is higher than that for $300 \mathrm{~ms}$, which is caused by the preheating when laser gets closer for the first time. Figure 5(c) shows the temperature distribution along the $z$ direction, the intersection of the symmetric plane and the plane with the $x$ coordinate of $2.25 \mathrm{~mm}$. The small figure shows the schematic for the gas phase, cladding bead and substrate. It can be seen that at time of
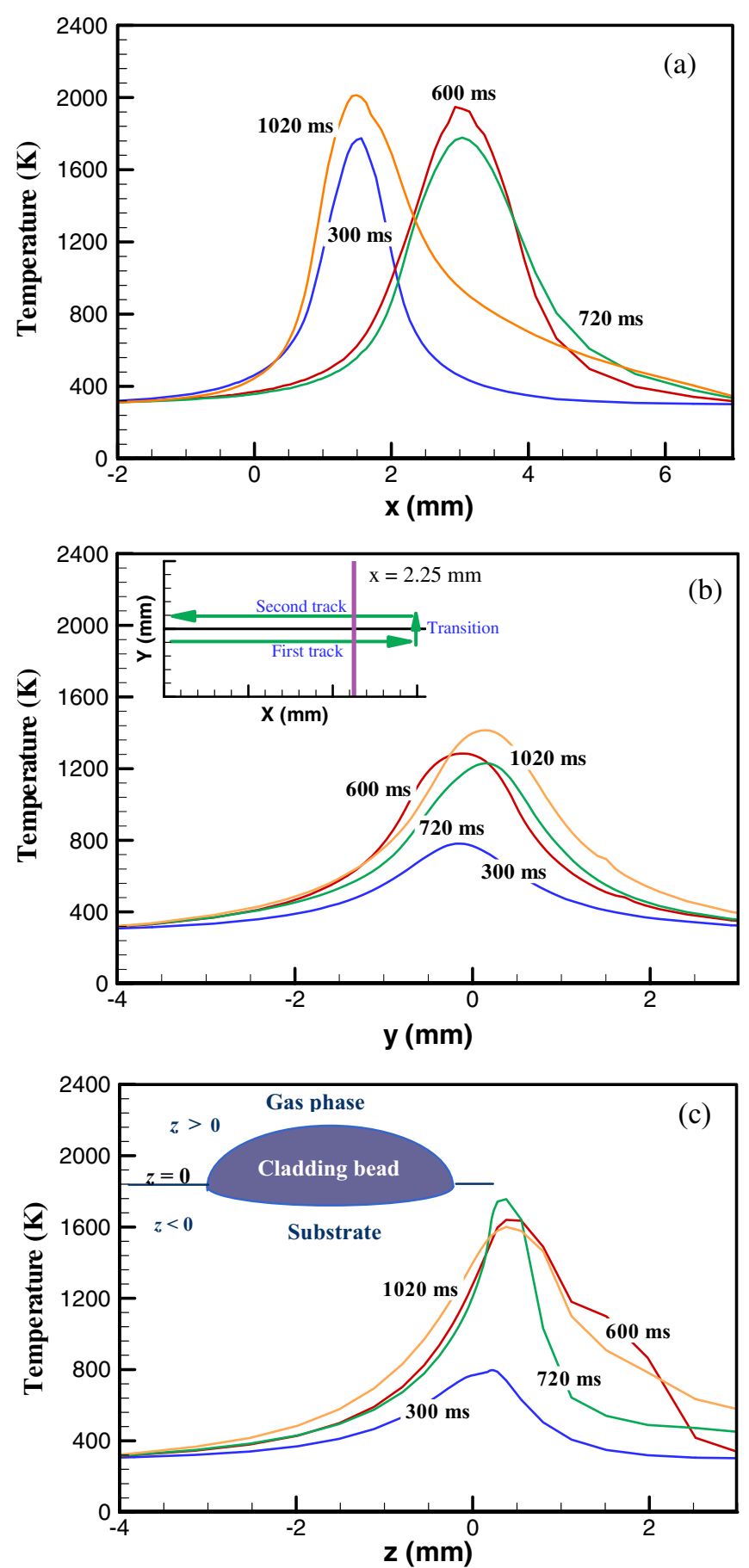

Figure 5. Temperature as a function of location along the (a) $x$, (b) $y$ and $(c) z$ direction at different times. Laser power: $1200 \mathrm{~W}$, beam diameter: $1.2 \mathrm{~mm}$, scanning speed: $300 \mathrm{~mm} \mathrm{~min}^{-1}$ and powder flow rate: $8 \mathrm{~g} \mathrm{~min}-1$.

$1020 \mathrm{~ms}$, the $z$ coordinate of the location with peak temperature is $0.34 \mathrm{~mm}$. In the calculation, not only preheating but also previously deposited mass from the first track is considered. With $75 \%$ overlap, the laser beam falls partly on the first track when returning. This is why the point with peak temperature is not located at the top surface.

3.2.4. Temperature and velocity fields of planes. Figure 6 shows the computed temperature and velocity fields at the top 

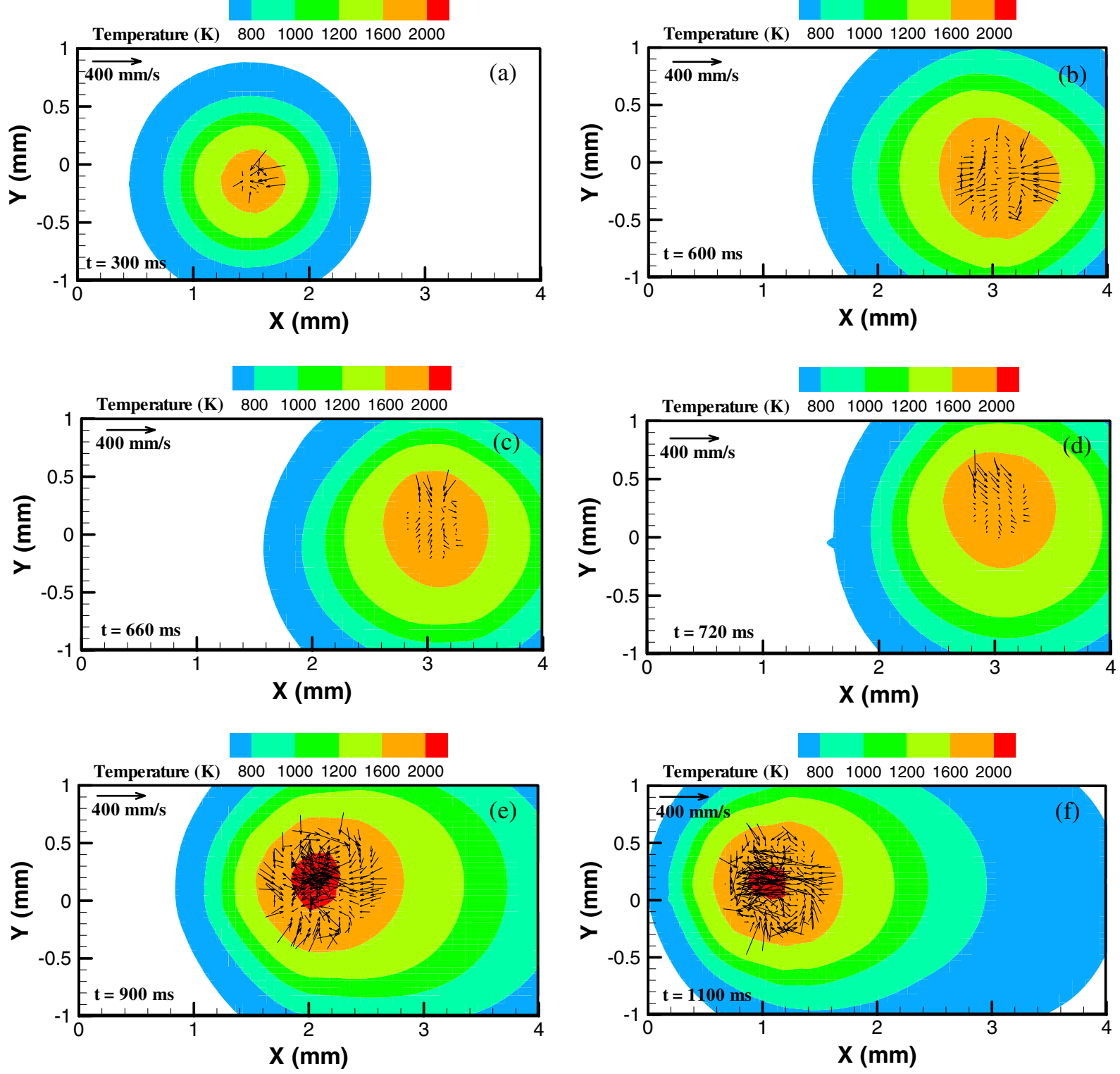

Figure 6. Computed temperature and velocity fields at the top surface at different times: $(a) t=300 \mathrm{~ms},(b) t=600 \mathrm{~ms},(c) t=660 \mathrm{~ms}$, $(d) t=720 \mathrm{~ms},(e) t=900 \mathrm{~ms}$ and $(f) t=1100 \mathrm{~ms}$. Laser power: $1200 \mathrm{~W}$, beam diameter: $1.2 \mathrm{~mm}$, scanning speed: $300 \mathrm{~mm} \mathrm{~min}^{-1}$ and powder flow rate: $8 \mathrm{~g} \mathrm{~min}^{-1}$.

surface at different times. The temperature and velocity of the liquid metal are indicated by the contours and black arrows, respectively. Vectors are drawn with the head of the vector positioned at the data point. The liquid flow during heating is driven by buoyancy, capillary and thermocapillary forces. The maximum velocity is about $1.7 \mathrm{~m} \mathrm{~s}^{-1}$ when the liquid pool is fully developed.

\subsection{The composition profile}

In laser cladding, species transportation in the liquid pool can be in the form of convection and diffusion. In past work, the dimensionless Peclet number, $P e$, was used to determine the relative importance of convection and conduction in the heat transfer in laser processing [29]. Similarly, the Peclet number for mass transfer, $P e_{m}$, can be used to indicate the relative importance of convection flow and solute diffusion in the overall transport of solute in the liquid pool [30].

$$
P e_{m}=\frac{u L_{R}}{D}
$$

where $u$ is the typical liquid velocity, $L_{R}$ is the characteristic length taken as the pool radius and $D$ is the mass diffusivity. The three parameters take the value of $1 \mathrm{~m} \mathrm{~s}^{-1}, 0.5 \mathrm{~mm}$ and $3.0 \times 10^{-8} \mathrm{~m}^{2} \mathrm{~s}^{-1}$, respectively, the corresponding value of $P e_{m}$ is calculated to be $1.7 \times 10^{4}$. Convection flow is the primary mechanism of mass transport in the liquid pool. Therefore, it is necessary to consider the convective mass transport in order to simulate the solute concentration distribution accurately.

Figures 7(a)-(e) show the computed concentration profile of carbon along the symmetric plane during double-track laser cladding at different times. The composition of alloying 

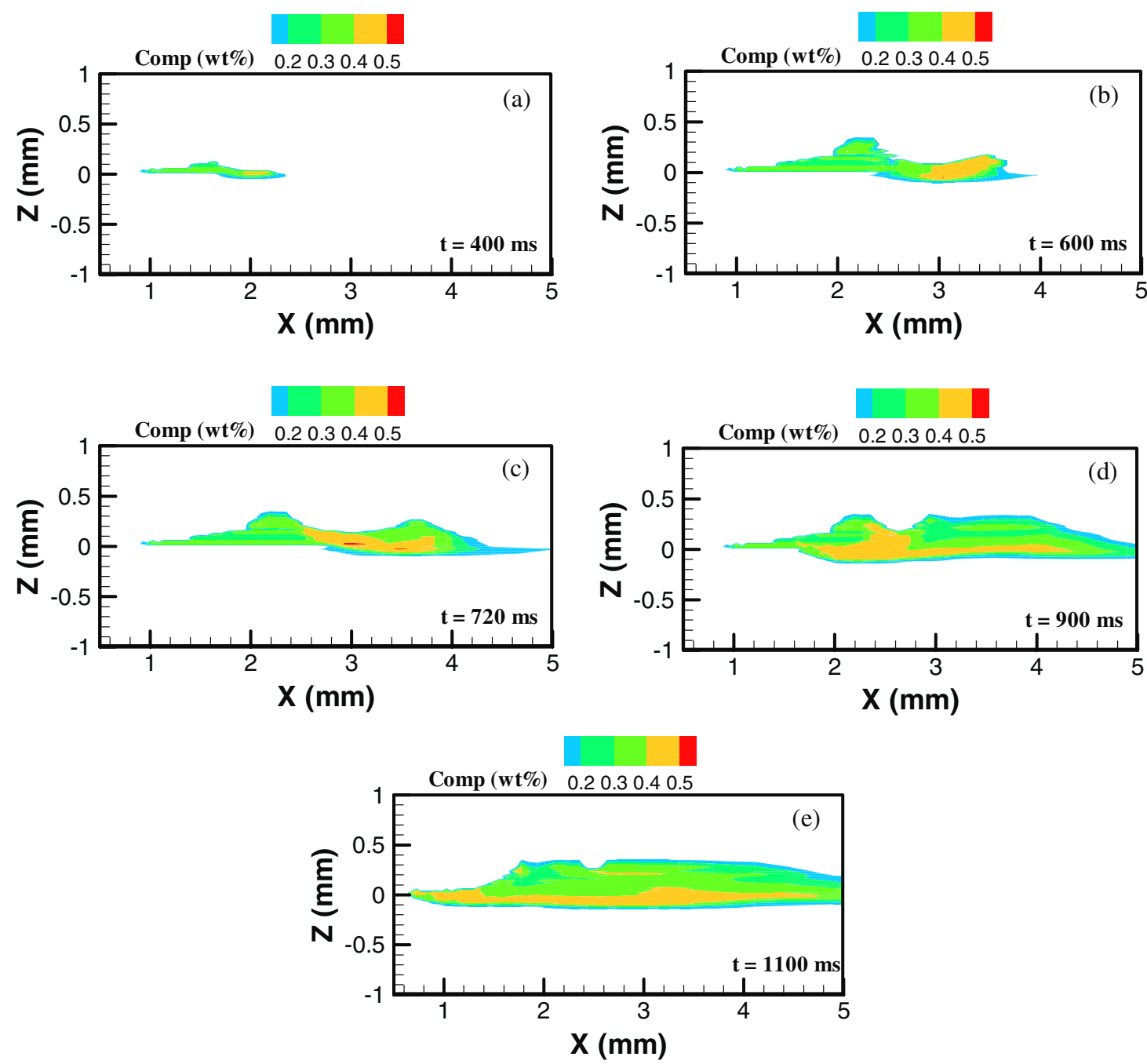

Figure 7. Computed concentration profile of carbon along the symmetric plane during double-track laser cladding at different times: (a) $t=300 \mathrm{~ms},(b) t=600 \mathrm{~ms},(c) t=720 \mathrm{~ms},(d) t=900 \mathrm{~ms}$ and $(e) t=1100 \mathrm{~ms}$. Laser power: $1200 \mathrm{~W}$, beam diameter: $1.2 \mathrm{~mm}$, scanning speed: $300 \mathrm{~mm} \mathrm{~min}^{-1}$ and powder flow rate: $8 \mathrm{~g} \mathrm{~min}^{-1}$.

Table 3. Composition of alloying elements in $\mathrm{H} 13$ powder (wt\%).

\begin{tabular}{lll}
\hline Element & Min. & Max. \\
\hline Carbon & 0.37 & 0.42 \\
Manganese & 0.2 & 0.5 \\
Phosphorus & 0 & 0.025 \\
Sulfur & 0 & 0.005 \\
Silicon & 0.2 & 0.8 \\
Chromium & 5 & 5.5 \\
Vanadium & 0.8 & 1.2 \\
Molybdenum & 1.2 & 1.75 \\
\hline
\end{tabular}

elements of $\mathrm{H} 13$ is shown in table 3. In the calculation, concentration of carbon in $\mathrm{H} 13$ powder is taken as $0.4 \mathrm{wt} \%$. At the time of $200 \mathrm{~ms}$, powder begins to penetrate into the low carbon steel substrate and a small dilution is formed. The diluted layer is contributed mainly by convection flow and to a much lesser extent by solute diffusion in the melt pool. As convection in the liquid pool gets stronger with time, the cladding depth increases until the liquid pool is fully developed. As a result, a good metallurgical bonding is formed between powder and substrate.

Figure 8 shows the computed concentration profile of carbon at the top surface when the liquid pool is fully developed. Although the movement track of laser beam is symmetrical with respect to the $y$-axis, the spatial distribution of concentration is not symmetrical. From the thermal cycle shown in figure 4, for two points symmetrical with respect to the $y$-axis, the point closer to the second track has higher peak temperature than the other point due to preheating. Besides preheating, previously deposited mass from the first track has an effect on the second track, which may result in the asymmetry of spatial concentration distribution. The cladding bead is wider for $y>0$. Therefore, if a symmetric concentration distribution is expected in real experiments, the adjustment of the processing parameter may be needed. A little deviation from symmetrical movement track of laser beam, a little lower laser scanning speed or higher laser power for the first track would be helpful. However, it should be noted that the concentration profile is simulated for only one set of laser 


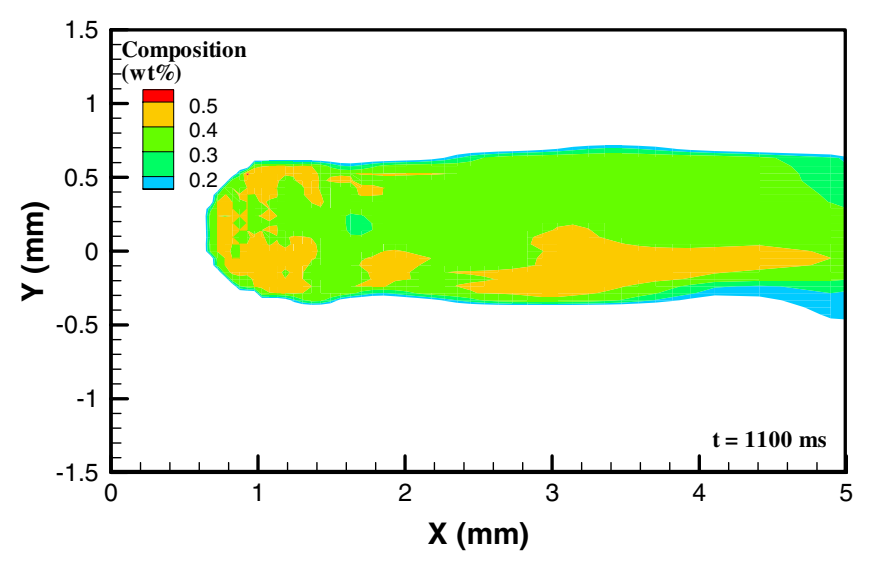

Figure 8. Computed concentration profile of carbon at the top surface during double-track laser cladding at the time of $1100 \mathrm{~ms}$. Laser power: $1200 \mathrm{~W}$, beam diameter: $1.2 \mathrm{~mm}$, scanning speed: $300 \mathrm{~mm} \mathrm{~min}^{-1}$ and powder flow rate: $8 \mathrm{~g} \mathrm{~min}^{-1}$.

processing parameters, the results may be used with caution for other processing parameters.

\section{Conclusions}

A comprehensive three-dimensional numerical model has been developed for the double-track laser cladding process of H13 tool steel. The temperature and composition distribution during the double-track laser cladding process is simulated based on the solutions of the equations of mass, momentum, energy conservation and solute transportation in the liquid pool, incorporating heat transfer, phase changes, mass addition and fluid flow. The laser beam velocity during the transition process between two tracks is considered in the calculation. Some of the important findings from the simulation are as follows:

(1) Both the preheating and previously deposited mass from the first track have effects on the second track. For the points with the similar geometric position, the peak temperature for the second track is higher than that for the first track, and the spatial concentration distributions are different.

(2) Under the selected laser processing parameters, the maximum temperature position along the $z$ direction for the second track is not located at the top surface, but at $z=0.34 \mathrm{~mm}$ due to the previously deposited mass from the first track.

(3) The maximum fluid velocity can reach as high as $1.7 \mathrm{~m} \mathrm{~s}^{-1}$ when the liquid pool is fully developed. The Peclet number for mass transfer is in the order of $10^{4}$, and the strong convective motion dominates the species transportation.

\section{Acknowledgment}

The work was supported by a grant from the Department of Commerce, Advanced Technology Program, under grant number 70NANB4H3027. Dr Jean Loui Steademann is the Program Manager. The authors would like to thank Dr Lijun Song for his valuable experimental work in POM.

\section{References}

[1] He X and Mazumder J 2007 J. Appl. Phys. 101053113

[2] Cho C D, Zhao G P, Kwak S Y and Kim C B 2004 J. Mater. Process. Technol. 153-154 494-500

[3] Kumar S, Roy S, Paul C P and Nath A K 2008 Numer. Heat Transfer B 53 271-87

[4] Kumar S and Roy S 2008 Comput. Mater. Sci. 41 457-66

[5] Toyserkani E, Khajepour A and Corbin S 2004 Opt. Lasers Eng. 41 849-67

[6] Liu C Y and Lin J N 2003 Opt. Laser Technol. 35 81-6

[7] Choi J, Han L and Hua Y 2005 J. Heat Transfer 127 978-86

[8] Han L, Liou F W and Phatak K M 2004 Metall. Mater. Trans. B 35 1139-50

[9] Han L, Phatak K M and Liou F W 2005 J. Laser Appl. 17 89-99

[10] Qi H, Mazumder J and Ki H 2006 J. Appl. Phys. 100024903

[11] Pinkerton A J and Li L 2004 J. Phys. D: Appl. Phys. 37 1885-95

[12] Colombo V, Mentrelli A and Trombetti T 2003 Eur. Phys. J. D 27 239-46

[13] Ghosh S and Choi J 2005 J. Laser Appl. 17 144-58

[14] Ghosh S and Choi J 2006 J. Heat Transfer 128 662-79

[15] Lakhkar, R S, Shin Y C and Krane M J M 2008 Mater. Sci. Eng. A 480 209-17

[16] Li Y M, Liu Z X, Yang H O, Lin X, Huang W D and Li J G 2003 Acta Metall. Sin. 39 521-25

[17] Costa L, Vilar R, Reti T, Colaco R, Deus A M and Felde I 2005 Mater. Sci. Forum 473-474 315-20

[18] Costa L, Vilar R, Reti T and Deus A M 2005 Acta Mater. 53 3987-99

[19] Jendrzejewski R, Kreja I and Sliwinski G 2004 Mater. Sci. Eng. A 379 313-20

[20] Jendrzejewski R, Sliwinski G, Krawczuk M and Ostachowicz W 2006 Surf. Coat. Technol. 201 3328-34

[21] Wang L and Felicelli S 2007 J. Heat Transfer 129 1028-34

[22] Kelly S M and Kampe S L 2004 Metall. Mater. Trans. A 35 1869-79

[23] Chande T and Mazumder J 1985 J. Appl. Phys. 57 2226-32

[24] Asai S and Muchi I 1978 Trans. Iron Steel Inst. Japan 18 90-8

[25] Bennon W I 1987 Int. J. Heat Mass Transfer 30 2160-70

[26] Bennon W I 1987 Int. J. Heat Mass Transfer 30 2171-87

[27] Vanka S 1986 J. Comput. Phys. 65 138-58

[28] Ki H, Mohanty P S and Mazumder J 2002 Metall. Mater. Trans. A 33 1817-30

[29] He X, Elmer J W and Debroy T 2005 J. Appl. Phys. 97084909

[30] Mishra S, Chakraborty S and DebRoy T 2005 J. Appl. Phys. 97094912 